\title{
Bird species distribution and conservation in Serra do Cipó, Minas Gerais, Brazil
}

\author{
TADEU A. MELO-JÚNIOR, MARCELO F. DE VASCONCELOS, GERALDO \\ W. FERNANDES and MIGUEL Â. MARINI
}

\begin{abstract}
Summary
We studied the distribution of birds along an altitudinal gradient ranging from $800 \mathrm{~m}$ to $1,400 \mathrm{~m}$ on two slopes of the Serra do Cipó, Minas Gerais, southeastern Brazil. Ornithological surveys were conducted over transects covering open (cerrado, campo rupestre) and forested (gallery and Atlantic forest) habitats from 1994 to 2000 . We found 273 bird species belonging to 51 families. Twenty-two species were restricted to higher elevations and 84 species were detected on only one slope, depending on the vegetation type they inhabited. We recorded 104 species occurring on both slopes, while 61 species were considered altitudinal generalists. Six species, including Hyacinth Visorbearer Augastes scutatus and Cipó Canastero Asthenes luizae were restricted to the highest parts of Serra do Cipó, a fragile habitat important to endemic birds of the Espinhaço Range. In the past 10 years, the Serra do Cipó region has suffered human impacts on a large scale, and conservation action must be developed to protect the fauna and flora confined to the area.
\end{abstract}

\section{Introduction}

Although the Brazilian bird fauna is one of the most diverse in the world (Sick 1997), studies involving patterns along altitudinal gradients are limited to Holt (1928), Scott and Brooke (1985), Bencke and Kindel (1999), Goerck (1999) and Buzzetti (2000). This is especially true for the Espinhaço region, a mountainous area in southeastern Brazil, which, even though a globally important centre of plant and animal endemism (Giulietti and Pirani 1988, Harley 1988, Barros 1990, Giulietti and Hensold 1990, Wanderley 1990, Eiten 1992, Silva 1995a, 1998, Giulietti et al. 1997, Sick 1997, Stattersfield et al. 1998), has not yet been studied to the extent it deserves. Within the Espinhaço Range vegetation composition and structure change dramatically along altitudinal gradients, offering a landscape where the influence of habitat complexity upon biological diversity can be studied in detail in a small geographical area. In addition, this mountain range separates two important biomes in its central and southern portion: the Atlantic forest on the eastern slope, and the cerrado on the western slope (Giulietti and Pirani 1988, Harley 1995, Giulietti et al. 1997). The highest parts of the Espinhaço Range consist of campo rupestre, a rupestrian grassland with many endemic plant taxa, growing among and on rocky outcrops, with scattered shrubs and small trees (Menezes and Giulietti 1986, 2000; Eiten 1992; Giulietti et al. 1997).

The mountainous Serra do Cipó, particularly the southern Espinhaço Range, 
lends itself to the study of species composition along altitudinal gradients and between slopes. Several studies on the distribution of insects and plants in Serra do Cipó have been carried out, in an attempt to better understand the effects of altitude (changes in temperature, moisture, and soil nutritional status) on patterns of species richness (Fernandes and Price 1988, Carneiro et al. 1995, Lara and Fernandes 1996, Ribeiro et al. 1998). The primary goal of this study was to describe bird species distribution along the altitudinal gradient of Serra do Cipó and between eastern (Atlantic Forest) and western (cerrado) slopes, to inform conservation action in the region.

\section{Study area}

Serra do Cipó is located in the central area of Minas Gerais State, southeastern Brazil, between $19^{\circ} 12^{\prime}-19^{\circ} 34^{\prime} \mathrm{S}$ and $43^{\circ} 27^{\prime}-43^{\circ} 38^{\prime} \mathrm{W}$, in the southern portion of the Espinhaço Range. The Serra do Cipó National Park comprises 33,800 ha (Figure 1). The altitudinal range is from $c$. 800 to $c$. 1,60o $\mathrm{m}$, with one peak (Pico do Breu) reaching $1,687 \mathrm{~m}$ (Goulart 2000). The climate is tropical montane with mean annual temperature varying between 17 and $18.5^{\circ} \mathrm{C}$, and mean annual precipitation between 1,450 and $1,850 \mathrm{~mm}$ (Antunes 1986). Precipitation falls primarily between December and March (Antunes 1986). Our study was conducted at the Serra do Cipó region, in the municipalities of Lagoa Santa, Jaboticatubas, Santana do Riacho, Itambé do Mato Dentro, Morro do Pilar, and Conceição do Mato Dentro and along the road (MG о10) that crosses the region (Figure 1).

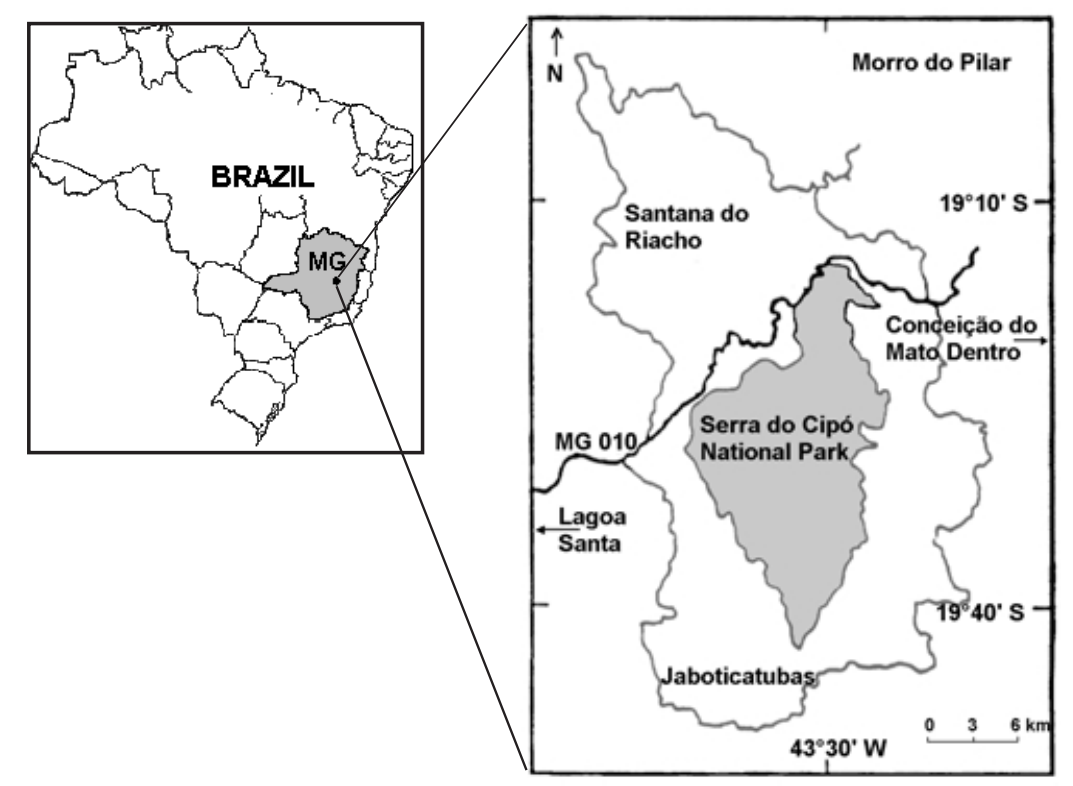

Figure 1. Map of Serra do Cipó region, Minas Gerais state, southeastern Brazil. Sites used in this study were situated in Serra do Cipó National Park, municipalities borders and along the road (MG о10). 


\section{Vegetation}

On the western slope, the vegetation between 800 and $1,000 \mathrm{~m}$ is cerrado, a savanna-like vegetation typical of central Brazil (Eiten 1992), intermixed with riparian and gallery forests that occur along water courses (Ribeiro and Walter 1998). At the base of the mountain there are small lakes, marshlands, plantations, and pastures. At $1,000 \mathrm{~m}$ a mixed cerrado-campo rupestre habitat is found, as well as small areas of riparian forest.

The Atlantic forest is the predominant vegetation on the eastern slope between 800 and $1,000 \mathrm{~m}$. At the base of the range there are small lakes, riverine habitats, pasture, and some plantations. At $1,000 \mathrm{~m}$, there is a transition from Atlantic forest to campos rupestres. A typical transition forest known as candeial is found in places, composed predominantly of candeia trees (Asteraceae: Eremanthus spp.).

Between $1,000 \mathrm{~m}$ and $1,300 \mathrm{~m}$ on both slopes, campo rupestre vegetation predominates, with narrow riparian forests also occurring. Above 1,300 m, a plateau of wide, humid open grasslands lacks the rocky outcrops found at lower elevations. Marshes and small patches of campos rupestres also occur, and there are narrow strips of riparian gallery forests and small natural forest fragments.

\section{Methods}

Bird surveys were carried out along an altitudinal gradient between $800 \mathrm{~m}$ and $1,400 \mathrm{~m}$ over 91 days distributed amongst all seasons and elevations, between 1994 and 2000. Birds were recorded along linear transects $1.5 \mathrm{~km}$ in length, in the morning (o6hoo to 10h30) and in the afternoon (14hoo to 18h30). Each transect was walked during a fixed time interval of about $1 \mathrm{~h}$. Bird species were identified by visual observation and by their song, using recordings (Sick 1979, 1997, Richards 1981). To provide a documented database of the regional avifauna, some birds were tape-recorded and/or photographed. We also searched specimens collected in the 1970s $(n=15)$, deposited in the Zoology Department of the Federal University of Minas Gerais (DZUFMG).

\section{Results}

Bird species composition and distribution

We recorded 273 bird species of 51 families in the Serra do Cipó region (Table 1). Most birds were Tyrannidae and Emberizidae $(n=98,35.9 \%)$. Ten $(3.6 \%)$ of the species were endemic to Brazil (Sick 1997), 15 (5.4\%) were endemic to the Cerrado region (Silva 1995a) and 14 (5.1\%) to the Atlantic forest (Sick 1997). Three $(1.4 \%)$ species were restricted to the campos rupestres of the Espinhaço Range (Silva 1995a, Stattersfield et al. 1998). Seven (2.5\%) species were globally near-threatened, and three (1.1\%) were threatened in Minas Gerais State (Collar et al. 1992, 1994, Machado et al. 1998).

Some interesting patterns in altitudinal and slope preferences of bird species emerged from this study (Appendix 1). On the western slope, 76 species $(27.8 \%$ ) occurred exclusively at the lowest altitudes, while on the eastern slope only seven species $(2.5 \%)$ were found exclusively at lower elevations. There were 104 (38.1\%) widespread species that occurred in both slopes, with six $(2.1 \%)$ found 
exclusively at higher elevations. A total of 31 species (11.4\%) were altitudinal generalists, found along the whole altitudinal gradient.

Noteworthy records are as follows:

King Vulture Sarcoramphus papa Recorded regularly at high elevations (1,100$1,400 \mathrm{~m}$ ). Immatures were also observed in three different years, indicating that Serra do Cipó could be a regular breeding site for this near-threatened species in Minas Gerais State.

Spot-tailed Nightjar Caprimulgus maculicaudus A single bird was heard at dusk on 18 October 1999 in a pasture close to a marsh at the base of Serra do Cipó. A male was observed at night by spotlight on 27 October 1999 on a dirt road in the same area.

Hyacinth Visorbearer Augastes scutatus This endemic hummingbird was observed several times in the campos rupestres of Serra do Cipó. Breeding appears to occur in the dry season, since we found the cup-shaped nest of this species in June 1999.

Cipó Canastero Asthenes luizae Small and isolated populations have been reported to the north of its previously known range in the Espinhaço mountains (Cordeiro et al. 1998). In this study it was regularly recorded in an unprotected area frequently visited by people, covering approximately $5 \mathrm{~km}$ of the highway MG 010 at the top of the Serra do Cipó.

Scytalopus sp. Two records were made between 1995 and 1997 in the Fazenda Cipó region. In addition, a handful of records have been made elsewhere in the Espinhaço range, e.g. Serra do Caraça and Serra da Piedade (TAMJ and MFV pers. obs.). Cited by some authors (e.g. Willis and Oniki 1991) as Scytalopus novacapitalis, a species known to occur in western and southwestern Serra do Cipó region, but although the plumage pattern is similar to that species, the vocalization pattern is closest to $S$. speluncae.

Helmeted Manakin Antilophia galeata Regularly recorded at 800-900 m, with a few records at 1,000-1,100 m, in gallery forests. Above 1,000 m, forest fragments were sparser and less connected, possibly presenting difficulties in dispersion of this species at higher altitudes.

Golden-Crowned Warbler Basileuterus culicivorus One of the most frequently recorded species, represented by two subspecies, Basileuterus culicivorus culicivorus and B. c. hypoleucos, occurring sympatrically at higher elevations. We also observed and tape-recorded a hybrid individual at 1,000 $\mathrm{m}$ altitude.

Black-headed Goldfinch Carduelis magellanica Restricted to 1,100-1,200 m altitude. According to local people, this species was commonly found in former times at lower altitudes, where the human population density is higher. Its 
absence below 1,000 $\mathrm{m}$ altitude could be linked to the pressure of capture for the cage bird trade.

\section{Discussion}

This study has shown that the Serra do Cipó region supports a high diversity of birds. Most species restricted to the western slope were typical birds of the cerrado vegetation (e.g. Crested Jay Cyanocorax cristatellus, Coal-crested Finch Charitospiza eucosma), while others were species associated with water courses or marshes (e.g. Cocoi Heron Ardea cocoi, Whistling Ducks Dendrocygna spp., South American Snipe Gallinago paraguaiae). Other cerrado birds (e.g. Chestnut-capped Foliage-gleaner Hylocryptus rectirostris, Helmeted Manakin Antilophia galeata) were also found in gallery forests. The species restricted to the eastern slope were Tataupa Tinamou Crypturellus tataupa, Striped Owl Rhynoptynx clamator, a unidentified Hermit Phaethornis sp., Tufted Antshrike Mackenziaena severa, Blue Manakin Chiroxiphia caudata, Pin-tailed Manakin Ilicura militaris and Violaceus Euphonia Euphonia violacea. Species widespread in the Serra do Cipó ranged from the Atlantic forest towards the Cerrado region, occurring in patches of forest (e.g. Serra Antwren Formicivora serrana, White-shouldered Fire-eye Pyriglena leucoptera, White-eyed Foliage-gleaner Automolus leucophthalmus).

Most bird species encountered in this study (91.2\%) were also recorded by Parrini et al. (1999) at Chapada Diamantina, a northern Espinhaço range extension at Bahia State. Our results showed a similarity between the fauna of Cerro San Simon, northeastern Bolivia (Parker and Rocha 1991) and that of Serra do Cipó, with the following bird species occurring in both regions: Red-winged Tinamou Rhynchotus rufescens, Pale-vented Pigeon Columba cayennensis, Horned Sungem Heliactin bilopha, White-eared Puffbird Nystalus chacuru, Collared Crescentchest Melanopareia torquata, Grey Monjita Xolmis cinerea, Black-throated Saltator Saltator atricollis, and Yellow-billed Blue-Finch Porphyrospiza caerulescens. However, all these species are widespread in the domain of the cerrado (see Silva 1995a, b). Interestingly, Chapada Diamantina and Cerro San Simon also have a flora similar to that of the Serra do Cipó.

This study offers an initial description of bird distribution in Serra do Cipó, which has been extensively studied by botanists and entomologists, but not ornithologists. Additional detailed studies are needed to test the hypotheses involving correlations between bird species richness, altitude and habitat structure in the Serra do Cipó region.

\section{Conservation}

During the last 10 years the Serra do Cipó region has been subjected to many threats resulting from the unplanned expansion of ecotourism, road construction, fires and other developments that contribute to habitat degradation. Even though the Atlantic forest is considered an important hotspot for biodiversity (Mittermeier et al. 1999), the remaining patches of the eastern slope have been largely destroyed by human activity. Forest fragmentation has had negative impacts on birds in Minas Gerais State, in both the Atlantic forest (Ribon 1998, Maldonado-Coelho and Marini 2000) and the cerrado biomes (Christiansen and 
Pitter 1997, Marini 2001). Despite being considered a high conservation priority area in Minas Gerais State due to its unique flora and fauna (Costa et al. 1998), there has been a lack of political effort devoted to its conservation.

Andrade (1999) showed that even small (<1 ha) natural forest patches similar to those occurring at Serra do Cipó, support a rich fauna of forest-dependent birds, demonstrating the importance of these forests to the conservation of birds living in the Brazilian highlands.

\section{Acknowledgments}

We would like to thank M. A. Pizo, M. Galetti, J. F. Pacheco, Bret M. Whitney, R. J. Donatelli, A. V. Christianini and two anonymous reviewers for their invaluable comments on earlier drafts of this manuscript. Special thanks to A.L.T. Souza for help to prepare the original project. This study could not have been accomplished without the logistic support provided by the Serra do Cipó National Park, Graduate Program in Ecology, Conservation and Wildlife Management/UFMG, Vellozia, and by the local people of Serra do Cipó. Field work was supported by grant provided by FAPEMIG (CBS 1385/95). TAM Jr. thanks FAPESP for financial support (97/04642-2). MFV received a grant from CAPES. GWF and MÂM received research fellowships from $\mathrm{CNPq}$. 
Appendix 1. Bird species $(n=273)$ recorded in the Serra do Cipó region, Minas Gerais, Brazil.

Key:

* Specimen deposited in the Zoology Depart., Universidade Federal de Minas Gerais.

Slope: W, western; E, eastern; T, top of the mountain.

Habitat types: 1, Atlantic forest fragments; 2, gallery forest or riparian forest; 3, small natural forest fragments on the mountaintop; 4, cerrado; 5, campo rupestre; 6, high montane humid field; 7, marsh; 8, lake; 9, area subject to human use.

Status and endemism (following Collar et. al. 1992, 1994, Silva 1995a, Sick 1997, Machado et. al. 1998): CE, endemic to cerrado $(n=15) ; \mathrm{AF}$, endemic to Atlantic forest $(n=14)$; ER, endemic to Espinhaço Range $(n=4)$; NT, Nearthreatened in Minas Gerais State $(n=7) ;$ MG, Threatened in Minas Gerais State $(n=3)$.

\begin{tabular}{|c|c|c|c|c|}
\hline Family/species & $\begin{array}{l}\text { Altitudinal } \\
\text { range }(\mathrm{m})\end{array}$ & Habitat & Slope & $\begin{array}{l}\text { Status and } \\
\text { endemism }\end{array}$ \\
\hline \multicolumn{5}{|l|}{ TINAMIDAE } \\
\hline Crypturellus parvirostris & $800-1,100$ & $4,5,9$ & W, E & \\
\hline Crypturellus tataupa & $900-1,000$ & $1,2,4,9$ & $\mathrm{E}$ & \\
\hline Rhynchotus rufescens & $800-1,100$ & 4,5 & W & \\
\hline Nothura maculosa & $800-1,400$ & $4^{-6}$ & $\mathrm{~W}, \mathrm{~T}$ & \\
\hline \multicolumn{5}{|l|}{ PODICIPEDIDAE } \\
\hline Tachybaptus dominicus & 800 & 8 & W & \\
\hline Podilymbus podiceps & 800 & 8 & W & \\
\hline \multicolumn{5}{|l|}{ PHALACROCORACIDAE } \\
\hline Phalacrocorax brasilianus & 800 & 8 & W & \\
\hline \multicolumn{5}{|l|}{ ANHINGIDAE } \\
\hline Anhinga anhinga & 800 & 8 & W & \\
\hline \multicolumn{5}{|l|}{ ARDEIDAE } \\
\hline Ardea cocoi & 800 & 8 & W & \\
\hline Casmerodius albus & 800 & 8 & W & \\
\hline Egretta thula & 800 & 8 & W & \\
\hline Bubulcus ibis & 800 & 8,9 & W & \\
\hline Butorides striatus & 800 & 7,8 & W, E & \\
\hline Syrigma sibilatrix & 800 & $4,7-9$ & W & \\
\hline Nycticorax nycticorax & 800 & 7,8 & W & \\
\hline \multicolumn{5}{|l|}{ THRESKIORNITHIDAE } \\
\hline Phimosus infuscatus & 800 & $4,7-9$ & W & \\
\hline \multicolumn{5}{|l|}{ CATHARTIDAE } \\
\hline Sarcoramphus papa & $1100-1,400$ & 5,6 & $\mathrm{~W}, \mathrm{~T}, \mathrm{E}$ & NT \\
\hline Coragyps atratus & $800-1,300$ & $1-5,9$ & $\mathrm{~W}, \mathrm{~T}, \mathrm{E}$ & \\
\hline Cathartes aura & $800-1,300$ & $1-5,9$ & $\mathrm{~W}, \mathrm{~T}, \mathrm{E}$ & \\
\hline \multicolumn{5}{|l|}{ ANATIDAE } \\
\hline Dendrocygna viduata & 800 & 7,8 & W, E & \\
\hline Dendrocygna autumnalis & 800 & 7,8 & W & \\
\hline Amazonetta brasiliensis & 800 & 7,8 & W & \\
\hline Cairina moschata & 800 & 7,8 & W & \\
\hline
\end{tabular}


Appendix 1. continued

\begin{tabular}{|c|c|c|c|c|}
\hline Family/species & $\begin{array}{l}\text { Altitudinal } \\
\text { range }(\mathrm{m})\end{array}$ & Habitat & Slope & $\begin{array}{l}\text { Status and } \\
\text { endemism }\end{array}$ \\
\hline \multicolumn{5}{|l|}{ ACCIPITRIDAE } \\
\hline Elanus leucurus & 800 & 4,9 & $\mathrm{~W}, \mathrm{E}$ & \\
\hline Rosthramus sociabilis & 800 & 8 & W & \\
\hline Geranoaetus melanoleucus & 1,100 & 5 & $\mathrm{~W}, \mathrm{E}$ & \\
\hline Buteo albonotatus & 1,000 & 4,5 & W & \\
\hline Buteo albicaudatus & 1,200 & 5 & $\mathrm{~W}, \mathrm{E}$ & \\
\hline Rupornis magnirostris & $800-1,400$ & $1-7,9$ & $\mathrm{~W}, \mathrm{~T}, \mathrm{E}$ & \\
\hline Geranospiza caerulescens & 800 & 2 & $\mathrm{~W}$ & \\
\hline Buteogallus meridionalis & 800 & 4,9 & $\mathrm{~W}, \mathrm{E}$ & \\
\hline \multicolumn{5}{|l|}{ FALCONIDAE } \\
\hline Herpetotheres cachinnans & 800 & $1,2,4,9$ & $\mathrm{~W}, \mathrm{E}$ & \\
\hline Micrastur semitorquatus & 800 & 2 & $\mathrm{~W}, \mathrm{E}$ & \\
\hline Milvago chimachima & $800-1,300$ & $1-7,9$ & $\mathrm{~W}, \mathrm{~T}, \mathrm{E}$ & \\
\hline Polyborus plancus & $800-1,000$ & $1-7,9$ & $\mathrm{~W}, \mathrm{E}$ & \\
\hline Falco femoralis & 8oo-900 & $1,2,4,9$ & $\mathrm{~W}, \mathrm{E}$ & \\
\hline Falco sparverius & $800-1,400$ & 5 & $\mathrm{~W}, \mathrm{~T}, \mathrm{E}$ & \\
\hline \multicolumn{5}{|l|}{ CRACIDAE } \\
\hline Penelope superciliaris & $800-1,000$ & 1,2 & $\mathrm{~W}, \mathrm{E}$ & \\
\hline \multicolumn{5}{|l|}{ ARAMIDAE } \\
\hline Aramus guarauna & 800 & 7,8 & W & \\
\hline \multicolumn{5}{|l|}{ RALLIDAE } \\
\hline Rallus nigricans & 800 & 7,8 & $\mathrm{~W}$ & \\
\hline Aramides cajanea & 800 & $1,2,4,7-9$ & $\mathrm{~W}, \mathrm{E}$ & \\
\hline Aramides saracura & $800-900$ & $1,2,7-9$ & $\mathrm{~W}, \mathrm{E}$ & $\mathrm{AF}$ \\
\hline Porzana albicollis & $800-900$ & 7,8 & $\mathrm{~W}, \mathrm{E}$ & \\
\hline Gallinula chloropus & 800 & 7,8 & $\mathrm{~W}$ & \\
\hline Porphyrula martinica & 800 & 7,8 & W & \\
\hline \multicolumn{5}{|l|}{ CARIAMIDAE } \\
\hline Cariama cristata & $800-1,300$ & $4-6,9$ & $\mathrm{~W}, \mathrm{~T}, \mathrm{E}$ & \\
\hline \multicolumn{5}{|l|}{ JACANIDAE } \\
\hline Jacana jacana & 800 & 7,8 & $\mathrm{~W}, \mathrm{E}$ & \\
\hline \multicolumn{5}{|l|}{ CHARADRIIDAE } \\
\hline Vanellus chilensis & 800 & 7-9 & $\mathrm{W}, \mathrm{E}$ & \\
\hline Charadrius collaris & 800 & 8 & $\mathrm{~W}$ & \\
\hline \multicolumn{5}{|l|}{ SCOLOPACIDAE } \\
\hline Tringa flavipes & 800 & 8 & W & \\
\hline Gallinago paraguaiae & $800-1,000$ & 7 & $\mathrm{~W}, \mathrm{E}$ & \\
\hline \multicolumn{5}{|l|}{ COLUMBIDAE } \\
\hline Columba livia domestica & $800-900$ & 9 & $\mathrm{~W}, \mathrm{E}$ & \\
\hline Columba picazuro & $800-1,200$ & $4,5,7,9$ & $\mathrm{~W}, \mathrm{E}$ & \\
\hline Columba cayennensis & $800-1,100$ & $4,5,9$ & $\mathrm{~W}, \mathrm{E}$ & \\
\hline Zenaida auriculata & 800 & 4,9 & W & \\
\hline Columbina talpacoti & $800-1,000$ & $4,5,9$ & $\mathrm{~W}, \mathrm{E}$ & \\
\hline Claravis pretiosa & 8 oot 2,9 & W & & \\
\hline Scardafella squammata & $800-1,000$ & $4,5,9$ & $\mathrm{~W}, \mathrm{E}$ & \\
\hline Leptotila verreauxi & $800-1,100$ & $1,2,4,9$ & $\mathrm{~W}, \mathrm{E}$ & \\
\hline Leptotila rufaxilla & 1,400 & 3 & $\mathrm{~T}$ & \\
\hline
\end{tabular}


Appendix 1. continued

\begin{tabular}{|c|c|c|c|c|}
\hline Family/species & $\begin{array}{l}\text { Altitudinal } \\
\text { range }(\mathrm{m})\end{array}$ & Habitat & Slope & $\begin{array}{l}\text { Status and } \\
\text { endemism }\end{array}$ \\
\hline \multicolumn{5}{|l|}{ PSITTACIDAE } \\
\hline Diopsittaca nobilis & 800 & $1,2,9$ & W & \\
\hline Aratinga leucophthalmus & 800 & $1,2,4,9$ & $\mathrm{~W}, \mathrm{E}$ & \\
\hline Aratinga aurea & $800-1,400$ & $4-6,9$ & $\mathrm{~W}, \mathrm{~T}$ & \\
\hline Pyrrhura frontalis & 800 & 1,2 & $\mathrm{~W}, \mathrm{E}$ & $\mathrm{NT}, \mathrm{AF}$ \\
\hline Forpus xanthopterygius & $800-1,200$ & $1,2,4-7,9$ & $\mathrm{~W}, \mathrm{E}$ & \\
\hline Brotogeris chiriri & $800-900$ & $2,4,9$ & W & \\
\hline Pionus maximiliani & $800-1,100$ & $1,2,4,9$ & W, E & \\
\hline Amazona aestiva & 800 & $2,4,9$ & $\mathrm{~W}$ & \\
\hline \multicolumn{5}{|l|}{ CUCULIDAE } \\
\hline Coccyzus americanus & 800 & 2,4 & W & \\
\hline Piaya cayana & $800-1,300$ & $1-3,9$ & $\mathrm{~W}, \mathrm{~T}, \mathrm{E}$ & \\
\hline Crotophaga ani & $800-1,000$ & $4,5,7,9$ & $\mathrm{~W}, \mathrm{E}$ & \\
\hline Guira guira & 800 & $4,5,7,9$ & $\mathrm{~W}, \mathrm{E}$ & \\
\hline Tapera naevia & $800-1,000$ & $1,2,4$ & $W, E$ & \\
\hline \multicolumn{5}{|l|}{ TYTONIDAE } \\
\hline Tyto alba & 800 & 4,9 & W & \\
\hline \multicolumn{5}{|l|}{ STRIGIDAE } \\
\hline Otus choliba & $800-900$ & $1,2,4,9$ & $\mathrm{~W}, \mathrm{E}$ & \\
\hline Rhynoptynx clamator & 900 & 4 & $\mathrm{E}$ & \\
\hline Pulsatrix perspicillata & 800 & 2 & $\mathrm{~W}$ & \\
\hline Glaucidium brasilianum & 800 & $1,2,4,9$ & $\mathrm{~W}, \mathrm{E}$ & \\
\hline Speotyto cunicularia & $800-1,400$ & $4,5,9$ & $\mathrm{~W}, \mathrm{~T}, \mathrm{E}$ & \\
\hline \multicolumn{5}{|l|}{ NYCTIBIIDAE } \\
\hline Nyctibius griseus & $800-1,400$ & $1-5$ & $\mathrm{~W}, \mathrm{~T}, \mathrm{E}$ & \\
\hline \multicolumn{5}{|l|}{ CAPRIMULGIDAE } \\
\hline Chordeiles pusillus & 800 & 4 & $\mathrm{~W}$ & \\
\hline Lurocalis semitorquatus & $800-900$ & $1,2,4$ & $\mathrm{~W}, \mathrm{E}$ & \\
\hline Nyctidromus albicollis & $800-1,000$ & $1,2,4,9$ & $\mathrm{~W}, \mathrm{E}$ & \\
\hline Caprimulgus rufus & 800 & $1,2,4$ & $\mathrm{~W}, \mathrm{E}$ & \\
\hline Caprimulgus longirostris & $1,000-1,300$ & 5 & $\mathrm{~W}, \mathrm{~T}, \mathrm{E}$ & \\
\hline Caprimulgus maculicaudus & 800 & 4,9 & $\mathrm{~W}$ & \\
\hline Caprimulgus parvulus & 800 & 4,9 & $\mathrm{~W}$ & \\
\hline Hydropsalis torquata & $900-1,100$ & $1,2,4,5,9$ & $\mathrm{~W}, \mathrm{E}$ & \\
\hline \multicolumn{5}{|l|}{ APODIDAE } \\
\hline Streptoprocne zonaris & $800-1,300$ & $1-6,9$ & $\mathrm{~W}, \mathrm{~T}, \mathrm{E}$ & \\
\hline Streptoprocne biscutata & 800 & 4,9 & $\mathrm{~W}, \mathrm{E}$ & \\
\hline Cypseloides fumigatus & 800 & 4,9 & $\mathrm{~W}$ & \\
\hline Chaetura andrei & $800-900$ & $1,2,4,9$ & $\mathrm{~W}, \mathrm{E}$ & \\
\hline \multicolumn{5}{|l|}{ TROCHILIDAE } \\
\hline Phaethornis pretrei * & $800-1,400$ & $1-5,7,9$ & $\mathrm{~W}, \mathrm{~T}, \mathrm{E}$ & \\
\hline Phaethornis sp. & 900 & 2 & E & \\
\hline Phaethornis ruber & 800 & 2 & $\mathrm{~W}$ & \\
\hline Eupetomena macroura & $800-1,200$ & $1,2,4,5,9$ & $\mathrm{~W}, \mathrm{E}$ & \\
\hline Colibri serrirostris * & $800-1,400$ & $4,5,9$ & $\mathrm{~W}, \mathrm{~T}, \mathrm{E}$ & \\
\hline Chlorostilbon aureoventris & $800-1,400$ & $1-5,9$ & $\mathrm{~W}, \mathrm{~T}, \mathrm{E}$ & \\
\hline Leucochloris albicollis & 1,300 & 3 & $\mathrm{~T}, \mathrm{E}$ & \\
\hline Amazilia lactea & $800-1,000$ & $1,2,4,5,9$ & $\mathrm{~W}, \mathrm{E}$ & \\
\hline Augastes scutatus * & $1,100-1,300$ & 5 & $\mathrm{~T}$ & $\mathrm{MG}, \mathrm{CE}, \mathrm{ER}$ \\
\hline Heliactin bilopha & $800-1,200$ & $4,5,9$ & W & \\
\hline
\end{tabular}


Appendix 1. continued

\begin{tabular}{|c|c|c|c|c|}
\hline Family/species & $\begin{array}{l}\text { Altitudinal } \\
\text { range }(m)\end{array}$ & Habitat & Slope & $\begin{array}{l}\text { Status and } \\
\text { endemism }\end{array}$ \\
\hline $\begin{array}{l}\text { TROGONIDAE } \\
\text { Trogon surrucura aurantius }\end{array}$ & $800-1,000$ & 1,2 & $\mathrm{~W}, \mathrm{E}$ & $\mathrm{AF}$ \\
\hline $\begin{array}{l}\text { ALCEDINIDAE } \\
\text { Ceryle torquata } \\
\text { Chloroceryle amazona } \\
\text { Chloroceryle americana }\end{array}$ & $\begin{array}{l}800-1,000 \\
800 \\
800\end{array}$ & $\begin{array}{l}8 \\
8 \\
8\end{array}$ & $\begin{array}{l}\text { W, E } \\
W \\
W\end{array}$ & \\
\hline $\begin{array}{l}\text { MOMOTIDAE } \\
\text { Baryphthengus ruficapillus }\end{array}$ & 800 & 2 & W & \\
\hline $\begin{array}{l}\text { GALBULIDAE } \\
\text { Galbula ruficauda }\end{array}$ & $800-1,000$ & 1,2 & W, E & \\
\hline $\begin{array}{l}\text { BUCCONIDAE } \\
\text { Nystalus chacuru } \\
\text { Nystalus maculatus } \\
\text { Malacoptila striata } \\
\text { Nonnula rubecula }\end{array}$ & $\begin{array}{l}800-1,300 \\
900 \\
800 \\
800\end{array}$ & $\begin{array}{l}4,5 \\
4 \\
1,2 \\
2\end{array}$ & $\begin{array}{l}\text { W, T, E } \\
W \\
W, E \\
W\end{array}$ & \\
\hline $\begin{array}{l}\text { RAMPHASTIDAE } \\
\text { Ramphastos toco }\end{array}$ & 800 & 4,9 & W & \\
\hline $\begin{array}{l}\text { PICIDAE } \\
\text { Picumnus cirratus } \\
\text { Colaptes campestris } \\
\text { Colaptes melanochloros } \\
\text { Dryocopus lineatus } \\
\text { Picoides mixtus } \\
\text { Melanerpes candidus } \\
\text { Veniliornis passerinus }\end{array}$ & $\begin{array}{l}800-1,200 \\
800-1,400 \\
800-1,100 \\
800-900 \\
900-1000 \\
800-900 \\
800-900\end{array}$ & $\begin{array}{l}1,2 \\
4,5,9 \\
1,2,4,9 \\
4 \\
4 \\
4,9 \\
1,2,4,9\end{array}$ & $\begin{array}{l}\text { W, T, E } \\
\text { W, T, E } \\
\text { W, E } \\
\text { W, E } \\
\text { W } \\
\text { W, E } \\
\text { W, E }\end{array}$ & \\
\hline $\begin{array}{l}\text { RHINOCRYPTIDAE } \\
\text { Melanopareia torquata } \\
\text { Scytalopus sp. }\end{array}$ & $\begin{array}{l}900-1,100 \\
1,000-1,100\end{array}$ & $\begin{array}{l}4,5 \\
3,5\end{array}$ & $\begin{array}{l}W \\
W\end{array}$ & $\begin{array}{l}\mathrm{CE} \\
\mathrm{CE}\end{array}$ \\
\hline $\begin{array}{l}\text { THAMNOPHILIDAE } \\
\text { Mackenziaena severa } \\
\text { Taraba major } \\
\text { Thamnophilus caerulescens } \\
\text { Thamnophilus torquatus } \\
\text { Dysithamnus mentalis } \\
\text { Herpsilochmus atricapillus } \\
\text { Formicivora serrana } \\
\text { Drymophila ochropyga } \\
\text { Pyriglena leucoptera }\end{array}$ & $\begin{array}{l}1,000 \\
800-900 \\
800-1,400 \\
1,000-1,200 \\
800 \\
800-900 \\
800-900 \\
1,300 \\
900\end{array}$ & $\begin{array}{l}1 \\
1,2,4,9 \\
1-3 \\
4,5 \\
1,2 \\
1,2 \\
1,2 \\
3 \\
1,2\end{array}$ & $\begin{array}{l}\text { E } \\
\text { W, E } \\
\text { W, T, E } \\
\text { W } \\
\text { W, E } \\
\text { W, E } \\
\text { W, E } \\
\text { T, E } \\
\text { W, E }\end{array}$ & $\begin{array}{l}\text { NT } \\
\text { AF } \\
\text { AF }\end{array}$ \\
\hline $\begin{array}{l}\text { FURNARIIDAE } \\
\text { Furnarius rufus } \\
\text { Furnarius figulus } \\
\text { Asthenes luizae } \\
\text { Synallaxis spixi * } \\
\text { Synallaxis ruficapilla } \\
\text { Synallaxis frontalis } \\
\text { Synallaxis albescens } \\
\text { Certhiaxis cinnamomea } \\
\text { Phacellodomus rufifrons }\end{array}$ & $\begin{array}{l}800-1,300 \\
800 \\
1,200 \\
800-1,400 \\
1,200-1,300 \\
800-1,400 \\
800-1,200 \\
800 \\
800-1,300\end{array}$ & $\begin{array}{l}4,5,9 \\
7,9 \\
5 \\
1-3,5 \\
3 \\
1-3,5 \\
4,5,7,9 \\
7,8 \\
4,5,9\end{array}$ & $\begin{array}{l}\text { W, T, E } \\
\text { W, E } \\
\text { T } \\
\text { W, T, E } \\
\text { T, E } \\
\text { W, T, E } \\
\text { W, E } \\
\text { W, E } \\
\text { W, T, E }\end{array}$ & $\mathrm{CE}, \mathrm{MG}, \mathrm{ER}$ \\
\hline
\end{tabular}


Appendix 1. continued

\begin{tabular}{|c|c|c|c|c|}
\hline Family/species & $\begin{array}{l}\text { Altitudinal } \\
\text { range }(\mathrm{m})\end{array}$ & Habitat & Slope & $\begin{array}{l}\text { Status and } \\
\text { endemism }\end{array}$ \\
\hline Anumbius annumbi & $800-1,100$ & $4,5,9$ & $\mathrm{~W}, \mathrm{E}$ & \\
\hline Automolus leucophthalmus & $800-900$ & 1,2 & $\mathrm{~W}, \mathrm{E}$ & $\mathrm{AF}$ \\
\hline Hylocryptus rectirostris & 800 & 2 & W & CE \\
\hline Xenops rutilans & 800 & 1,2 & $\mathrm{~W}, \mathrm{E}$ & \\
\hline Lochmias nematura & $800-1,300$ & $1-3,5$ & $\mathrm{~W}, \mathrm{~T}, \mathrm{E}$ & \\
\hline \multicolumn{5}{|l|}{ DENDROCOLAPTIDAE } \\
\hline Sittasomus griseicapillus & $800-1,000$ & 1,2 & $\mathrm{~W}, \mathrm{E}$ & \\
\hline Lepidocolaptes angustirostris & $800-1,100$ & $4,5,9$ & $\mathrm{~W}, \mathrm{E}$ & \\
\hline Lepidocolaptes fuscus & 800 & 1,2 & $\mathrm{~W}, \mathrm{E}$ & \\
\hline \multicolumn{5}{|l|}{ TYRANNIDAE } \\
\hline Phyllomyias fasciatus & $800-1,000$ & $1,2,4,5,9$ & $\mathrm{~W}, \mathrm{E}$ & \\
\hline Camptostoma obsoletum & $800-1,300$ & $1,2,4,5,9$ & $\mathrm{~W}, \mathrm{~T}, \mathrm{E}$ & \\
\hline Phaeomyias murina & $800-1,000$ & $1,2,4,5,9$ & W, E & \\
\hline Myiopagis caniceps & 900 & 2 & W & \\
\hline Myiopagis viridicata & $800-900$ & 1,2 & $\mathrm{~W}, \mathrm{E}$ & \\
\hline Elaenia flavogaster * & $800-1,100$ & $1,2,4,5,7,9$ & $\mathrm{~W}, \mathrm{E}$ & \\
\hline Elaenia mesoleuca & 800 & 4 & $\mathrm{~W}, \mathrm{E}$ & \\
\hline Elaenia cristata & $800-1,300$ & 4,5 & W & \\
\hline Elaenia obscura * & $1,000-1,100$ & $1,2,5$ & $\mathrm{~W}, \mathrm{E}$ & \\
\hline Elaenia chiriquensis & $800-1,300$ & 4,5 & $\mathrm{~W}, \mathrm{~T}$ & \\
\hline Serpophaga subcristata & $1,000-1,400$ & $1-3$ & $\mathrm{~W}, \mathrm{~T}, \mathrm{E}$ & \\
\hline Polystictus superciliaris & $1,100-1,300$ & 5 & $\mathrm{~W}, \mathrm{~T}, \mathrm{E}$ & NT, CE \\
\hline Leptopogon amaurocephalus & $800-1,400$ & $1-3$ & $\mathrm{~W}, \mathrm{~T}, \mathrm{E}$ & \\
\hline Phylloscartes ventralis & $900-1,300$ & 2,3 & $\mathrm{~T}, \mathrm{E}$ & $\mathrm{AF}$ \\
\hline Corythopis delalandi & 800 & 2 & W & \\
\hline Hemitriccus nidipendulus & $800-1,200$ & 1,2 & $\mathrm{~W}, \mathrm{E}$ & \\
\hline Todirostrum poliocephalum & $800-900$ & $1,2,9$ & $\mathrm{~W}, \mathrm{E}$ & $\mathrm{AF}$ \\
\hline Todirostrum plumbeiceps & 1,300 & 3 & $\mathrm{~T}, \mathrm{E}$ & \\
\hline Todirostrum latirostre & 800 & 2,4 & W & \\
\hline Tolmomyias sulphurescens & $800-1,400$ & $1-3$ & $\mathrm{~W}, \mathrm{~T}, \mathrm{E}$ & \\
\hline Platyrinchus mystaceus & 800 & 1,2 & $\mathrm{~W}, \mathrm{E}$ & \\
\hline Myiophobus fasciatus & $800-1,300$ & $4,5,7,9$ & $\mathrm{~W}, \mathrm{~T}, \mathrm{E}$ & \\
\hline Contopus cinereus & 800 & 2,4 & W & \\
\hline Lathrotriccus euleri & 800 & 1,2 & $\mathrm{~W}, \mathrm{E}$ & \\
\hline Cnemotriccus fuscatus bimaculatus & $800-900$ & 1,2 & $\mathrm{~W}, \mathrm{E}$ & \\
\hline Xolmis cinerea & $800-1,400$ & $4,5,9$ & $\mathrm{~W}, \mathrm{~T}, \mathrm{E}$ & \\
\hline Xolmis velata * & $800-1,100$ & $4,5,9$ & $\mathrm{~W}, \mathrm{E}$ & \\
\hline Knipolegus lophotes & $800-1,300$ & $4,5,9$ & W, T, E & \\
\hline Knipolegus nigerrimus * & $900-1,300$ & 4,5 & W, T, E & $\mathrm{AF}$ \\
\hline Knipolegus sp. & 1,300 & 5 & $\mathrm{~T}$ & \\
\hline Fluvicola nengeta & 800 & $7-9$ & $\mathrm{~W}, \mathrm{E}$ & \\
\hline Arundinicola leucocephala & 800 & 7,8 & $\mathrm{~W}, \mathrm{E}$ & \\
\hline Colonia colonus & $800-900$ & 4,9 & $\mathrm{~W}, \mathrm{E}$ & \\
\hline Gubernetes yetapa & 800 & 7 & $\mathrm{~W}, \mathrm{E}$ & \\
\hline Satrapa icterophrys & $800-900$ & 9 & $\mathrm{~W}, \mathrm{E}$ & \\
\hline Hirundinea ferruginea & $800-1,000$ & 4,5 & $\mathrm{~W}, \mathrm{E}$ & \\
\hline Machetornis rixosus & $800-900$ & 4,9 & $\mathrm{~W}, \mathrm{E}$ & \\
\hline Muscipipra vetula & $1,100-1,300$ & 3 & $\mathrm{~T}, \mathrm{E}$ & \\
\hline Casiornis rufa & 800 & 2 & W & \\
\hline Sirystes sibilator & 800 & 1,2 & W, E & \\
\hline Myiarchus ferox & $800-1,400$ & $1-5,9$ & $\mathrm{~W}, \mathrm{~T}, \mathrm{E}$ & \\
\hline Myiarchus tyrannulus & $800-900$ & $1,2,9$ & $\mathrm{~W}, \mathrm{E}$ & \\
\hline
\end{tabular}


Appendix 1. continued

\begin{tabular}{|c|c|c|c|c|}
\hline Family/species & $\begin{array}{l}\text { Altitudinal } \\
\text { range }(\mathrm{m})\end{array}$ & Habitat & Slope & $\begin{array}{l}\text { Status and } \\
\text { endemism }\end{array}$ \\
\hline $\begin{array}{l}\text { Pitangus sulphuratus } \\
\text { Megarhynchus pitangua } \\
\text { Myiozetetes similis } \\
\text { Myiodynastes maculatus } \\
\text { Empidonomus varius } \\
\text { Griseotyrannus aurantioatrocristatus } \\
\text { Tyrannus savana } \\
\text { Tyrannus melancholicus } \\
\text { Tyrannus albogularis } \\
\text { Pachyramphus viridis } \\
\text { Pachyramphus polychopterus } \\
\text { Pachyramphus validus } \\
\text { Tityra cayana }\end{array}$ & $\begin{array}{l}800-1,200 \\
800-1,000 \\
800-900 \\
800 \\
800-900 \\
800 \\
800-1,100 \\
800-1,100 \\
800 \\
800 \\
800-900 \\
800 \\
800\end{array}$ & $\begin{array}{l}1,2,4,5,9 \\
1,2,4,9 \\
1,2,4,9 \\
1,2,4,9 \\
1,2,4,9 \\
4 \\
1,2,4,9 \\
1,2,4,5,7,9 \\
4 \\
2 \\
1,2 \\
2 \\
2\end{array}$ & $\begin{array}{l}\text { W, E } \\
\text { W, E } \\
\text { W, E } \\
\text { W, E } \\
\text { W, E } \\
\text { W } \\
\text { W, E } \\
\text { W, E } \\
\text { W } \\
\text { W } \\
\text { W, E } \\
\text { W } \\
\text { W }\end{array}$ & \\
\hline $\begin{array}{l}\text { PIPRIDAE } \\
\text { Antilophia galeata } \\
\text { Chiroxiphia caudata } \\
\text { Ilicura militaris } \\
\text { Neopelma pallescens }\end{array}$ & $\begin{array}{l}800-1,100 \\
800-1,000 \\
900 \\
800\end{array}$ & $\begin{array}{l}2 \\
1 \\
1 \\
2\end{array}$ & $\begin{array}{l}W \\
E \\
E \\
W\end{array}$ & $\begin{array}{l}\mathrm{CE} \\
\mathrm{AF}\end{array}$ \\
\hline $\begin{array}{l}\text { COTINGIDAE } \\
\text { Pyroderus scutatus }\end{array}$ & 1,300 & 3 & $\mathrm{~T}, \mathrm{E}$ & $\mathrm{MG}, \mathrm{AF}$ \\
\hline $\begin{array}{l}\text { HIRUNDINIDAE } \\
\text { Tachycineta leucorrhoa } \\
\text { Notiochelidon cyanoleuca } \\
\text { Alopochelidon fucata } \\
\text { Stelgidopteryx ruficollis }\end{array}$ & $\begin{array}{l}800 \\
800-1,000 \\
1,200-1,400 \\
800-1,200\end{array}$ & $\begin{array}{l}2,8 \\
1,2,4,5,7-9 \\
5,6 \\
4-7,9\end{array}$ & $\begin{array}{l}\mathrm{W} \\
\mathrm{W}, \mathrm{E} \\
\mathrm{T} \\
\mathrm{W}, \mathrm{E}\end{array}$ & \\
\hline $\begin{array}{l}\text { CORVIDAE } \\
\text { Cyanocorax cristatellus }\end{array}$ & $800-1,300$ & $4,5,9$ & $\mathrm{~W}, \mathrm{~T}, \mathrm{E}$ & $\mathrm{CE}$ \\
\hline $\begin{array}{l}\text { TROGLODYTIDAE } \\
\text { Donacobius atricapillus } \\
\text { Troglodytes aedon }\end{array}$ & $\begin{array}{l}800 \\
800-1,300\end{array}$ & $\begin{array}{l}7,8 \\
1-7,9\end{array}$ & $\begin{array}{l}\text { W, E } \\
\text { W, T, E }\end{array}$ & \\
\hline $\begin{array}{l}\text { MUSCICAPIDAE } \\
\text { Turdus rufiventris } \\
\text { Turdus leucomelas } \\
\text { Turdus amaurochalinus }\end{array}$ & $\begin{array}{l}800-1,400 \\
800-1,400 \\
800-1,400\end{array}$ & $\begin{array}{l}1-5,9 \\
1-5,9 \\
1-5,9\end{array}$ & $\begin{array}{l}\text { W, T, E } \\
\text { W, T, E } \\
W, T, E\end{array}$ & \\
\hline $\begin{array}{l}\text { MIMIDAE } \\
\text { Mimus saturninus }\end{array}$ & $800-1,300$ & $4,5,9$ & $\mathrm{~W}, \mathrm{~T}, \mathrm{E}$ & \\
\hline $\begin{array}{l}\text { MOTACILLIDAE } \\
\text { Anthus cf hellmayri * } \\
\text { Anthus lutescens }\end{array}$ & $\begin{array}{l}1,400 \\
800\end{array}$ & $\begin{array}{l}5,6 \\
4,7-9\end{array}$ & $\begin{array}{l}\mathrm{T} \\
\mathrm{W}\end{array}$ & NT \\
\hline $\begin{array}{l}\text { VIREONIDAE } \\
\text { Cyclarhis gujanensis } \\
\text { Vireo chivi } \\
\text { Hylophilus amaurocephalus }\end{array}$ & $\begin{array}{l}800-1,400 \\
800-900 \\
800-900\end{array}$ & $\begin{array}{l}1-3,5 \\
1,2 \\
1,2\end{array}$ & $\begin{array}{l}\text { W, T, E } \\
\text { W, E } \\
\text { W, E }\end{array}$ & \\
\hline $\begin{array}{l}\text { EMBERIZIDAE } \\
\text { Parula pitiayumi } \\
\text { Geothlypis aequinoctialis } \\
\text { Basileuterus flaveolus } \\
\text { Basileuterus culicivorus culicivorus }\end{array}$ & $\begin{array}{l}800-900 \\
800-1,300 \\
800-1,100 \\
1,000-1,400\end{array}$ & $\begin{array}{l}2 \\
5-7,9 \\
1,2 \\
1-3\end{array}$ & $\begin{array}{l}\text { W } \\
\text { W, T, E } \\
\text { W, E } \\
\text { W, T, E }\end{array}$ & \\
\hline
\end{tabular}


Appendix 1. continued

\begin{tabular}{|c|c|c|c|c|}
\hline Family/species & $\begin{array}{l}\text { Altitudinal } \\
\text { range }(\mathrm{m})\end{array}$ & Habitat & Slope & $\begin{array}{l}\text { Status and } \\
\text { endemism }\end{array}$ \\
\hline Basileuterus culicivorus hypoleucus & $800-1,400$ & $1-3$ & W, T, E & \\
\hline Coereba flaveola & $800-1,400$ & $1-5,9$ & $\mathrm{~W}, \mathrm{~T}, \mathrm{E}$ & \\
\hline Schistochlamys ruficapillus * & $800-1,300$ & $4-6$ & $\mathrm{~W}, \mathrm{~T}, \mathrm{E}$ & \\
\hline Neothraupis fasciata & $800-900$ & 4 & W & CE \\
\hline Cypsnagra hirundinacea & 1,100 & 5 & W & NT, CE \\
\hline Hemithraupis ruficapilla & $800-1,300$ & $1-3$ & W, T, E & $\mathrm{AF}$ \\
\hline Nemosia pileata & $800-900$ & 2,4 & W & \\
\hline Piranga flava & $800-1,400$ & 2,5 & $\mathrm{~W}, \mathrm{~T}, \mathrm{E}$ & \\
\hline Thraupis sayaca & $800-1,400$ & $1-5,9$ & W, T, E & \\
\hline Euphonia violacea & 800 & & $\mathrm{E}$ & \\
\hline Euphonia chlorotica & $800-1,300$ & $1-5,9$ & $\mathrm{~W}, \mathrm{~T}, \mathrm{E}$ & \\
\hline Tangara cayana & $800-1,400$ & $1-5,9$ & W, T, E & \\
\hline Dacnis cayana & $800-1,000$ & $1,2,9$ & $\mathrm{~W}, \mathrm{E}$ & \\
\hline Conirostrum speciosum & 800 & 1,2 & $\mathrm{~W}, \mathrm{E}$ & \\
\hline Tersina viridis & $800-900$ & 2 & W & \\
\hline Zonotrichia capensis & $800-1,400$ & $4-6,9$ & $\mathrm{~W}, \mathrm{~T}, \mathrm{E}$ & \\
\hline Ammodramus humeralis * & $800-1,400$ & $4-6,9$ & $\mathrm{~W}, \mathrm{~T}, \mathrm{E}$ & \\
\hline Sicalis citrina * & $800-1,400$ & $4,5,9$ & $\mathrm{~W}, \mathrm{~T}, \mathrm{E}$ & \\
\hline Sicalis luteola & 800 & 9 & W & \\
\hline Emberizoides herbicola & $800-1,300$ & $6,7,9$ & $\mathrm{~W}, \mathrm{~T}, \mathrm{E}$ & \\
\hline Embernagra longicauda * & $1,000-1,400$ & 5 & $\mathrm{~W}, \mathrm{~T}, \mathrm{E}$ & CE, ER \\
\hline Volatinia jacarina & $800-1,100$ & $4,5,9$ & $\mathrm{~W}, \mathrm{E}$ & \\
\hline Sporophila nigricollis & $800-1,300$ & $4-6,9$ & $\mathrm{~W}, \mathrm{~T}, \mathrm{E}$ & \\
\hline Sporophila caerulescens & $800-1,100$ & $4,5,9$ & W, E & \\
\hline Sporophila bouvreuil bouvreuil & 800 & 7 & W & \\
\hline Arremon flavirostris & 800 & 2 & W & \\
\hline Charitospiza eucosma & 900 & 4 & W & NT, CE \\
\hline Coryphospingus pileatus & $800-1,400$ & $4,5,7,9$ & $\mathrm{~W}, \mathrm{~T}, \mathrm{E}$ & \\
\hline Saltator similis & $800-1,400$ & $1-3$ & $\mathrm{~W}, \mathrm{~T}, \mathrm{E}$ & \\
\hline Saltator atricollis * & $900-1,300$ & 4,5 & $\mathrm{~W}, \mathrm{~T}, \mathrm{E}$ & $\mathrm{CE}$ \\
\hline Passerina brissonii & $800-1,000$ & $4,7,9$ & $\mathrm{~W}, \mathrm{E}$ & \\
\hline Porphyrospiza caerulescens * & $800-1,300$ & 4,5 & $\mathrm{~W}, \mathrm{~T}$ & $\mathrm{CE}$ \\
\hline Psarocolius decumanus & 800 & $1,2,4,9$ & $\mathrm{~W}, \mathrm{E}$ & \\
\hline Cacicus haemorrhous & 800 & 1,2 & $\mathrm{~W}, \mathrm{E}$ & $\mathrm{AF}$ \\
\hline Agelaius ruficapillus & 800 & 7,8 & W & \\
\hline Leistes superciliaris & 800 & 7,9 & W & \\
\hline Pseudoleistes guirahuro & $1,200-1,400$ & $2,5,6$ & $\mathrm{~W}, \mathrm{~T}$ & \\
\hline Gnorimopsar chopi & $800-1,400$ & $4-6,9$ & W, T, E & \\
\hline Molothrus bonariensis & $800-1,200$ & $4,5,9$ & $\mathrm{~W}, \mathrm{E}$ & \\
\hline \multicolumn{5}{|l|}{ FRINGILLIDAE } \\
\hline Carduelis magellanicus & $1,100-1,200$ & 5 & W & \\
\hline \multicolumn{5}{|l|}{ PASSERIDAE } \\
\hline Passer domesticus & $800-900$ & 9 & $\mathrm{~W}, \mathrm{E}$ & \\
\hline
\end{tabular}




\section{References}

Andrade, R. D. (1999) Deslocamento e uso de capões de mata por aves do Parque Nacional da Serra da Canastra, Minas Gerais. M.Sc. Thesis, Universidade Federal de Minas Gerais, Belo Horizonte.

Antunes, F. Z. (1986) Caracterização climática do estado de Minas Gerais. Inf. Agropec. 12: $9-13$.

Barros, F. (1990) Diversidade taxonômica e distribuição geográfica das Orchidaceae brasileiras. Acta Bot. Bras. 4: 177-187.

Bencke, G. A. and Kindel, A. (1999) Bird counts along an altitudinal gradient of Atlantic forest in northeastern Rio Grande do Sul, Brazil. Ararajuba 7: 91-107.

Buzzetti, D. R. C. (2000) Distribuição altitudinal de aves em Angra dos Reis e Parati, Sul do Estado do Rio de Janeiro, Brasil. In: M. A. S. Alves, J. M. C. Silva, M. Van-Sluys, H. G. Bergallo and C. F. D. Rocha, eds. A ornitologia no Brasil: pesquisa atual e perspectivas. Rio de Janeiro: Editora da Universidade do Estado do Rio de Janeiro.

Carneiro M. A. A., Ribeiro, S. P. and Fernandes, G. W. (1995) Artrópodos de um gradiente altitudinal na Serra do Cipó, Minas Gerais, Brasil. Rev. Bras. Entomol. 39: $597-604$.

Christiansen, M. B. and Pitter, E. (1997) Species loss in a forest bird community near Lagoa Santa in southeastern Brazil. Biol. Conserv. 80: 23-32.

Collar, N. J., Gonzaga, L. P., Krabbe, N. Madroño Nieto, A., Naranjo, L. G., Parker III, T. A. and Wege, D. (1992) Threatened birds of the Americas: the ICBP/IUCN Red Data Book. Cambridge, U.K.: International Council for Bird Preservation.

Collar, N. J., Crosby, M. J. and Stattersfield, A. J. (1994) Birds to watch 2: the world list of threatened birds. Cambridge, U.K.: BirdLife International (Conservation Series 4).

Cordeiro, P.H., Melo-Júnior, T. A. and Vasconcelos, M.F. (1998) A range extension for Cipó Canastero, Asthenes luizae, in Brazil. Cotinga 10: 64-65.

Costa, C. M. R., Herrmann, G., Martins, C. S., Lins, L. V. and Lamas, I. R. (1998) Biodiversidade em Minas Gerais: um atlas para sua conservação. Belo Horizonte: Fundação Biodiversitas.

Eiten, G. (1992) Natural Brazilian vegetation types and their causes. An. Acad. Bras. Ci. 64: $35-65$.

Fernandes, G. W. and Price, P. W. (1988) Biogeographical gradients in galling species richness: tests of hypotheses. Oecologia 76: 161-167.

Giulietti, A. M. and Hensold, N. (1990) Padrões de distribuição geográfica dos gêneros de Eriocaulaceae. Acta Bot. Bras. 4: 133-158.

Giulietti, A. M. and Pirani, J. R. (1988) Patterns of geographic distribution of some plant species from the Espinhaço Range, Minas Gerais and Bahia, Brazil. In P. E. Vanzolini and W. R. Heyer, eds. Proceedings of a workshop on Neotropical distribution patterns. Rio de Janeiro: Academia Brasileira de Ciências.

Giulietti, A. M., Pirani, J. R. and Harley, R. M. (1997) Espinhaço Range region, Eastern Brazil. In S. D. Davis, V. H. Heywood, O. Herrera-MacBryde, J. Villa-Lobos and A. C. Hamilton, eds. Centres of plant diversity: a guide and strategy for their conservation, 3. Oxford: Information Press.

Goerck, J. (1999) Distribution of birds along an elevational gradient in an Atlantic forest remnant of Brazil: implications for the conservation of endemic and endangered species. Bird Cons. Internatn. 9:235-253.

Goulart, E. M. A. (2000) Nos ermos e nas brumas da Serra do Espinhaço. Belo Horizonte: Cooperativa Editora e de Cultura Médica.

Harley, R. M. (1988) Evolution and distribution of Eriope (Labiatae) and its relatives, in Brazil. In P. E. Vanzolini and W. R. Heyer, eds. Proceedings of a workshop on Neotropical distribution patterns. Rio de Janeiro: Academia Brasileira de Ciências. 
Harley, R. M. (1995) Introduction. In B. L. Stannard, Y. B. Harvey and R. M. Harley, eds. Flora of the Pico das Almas, Chapada Diamantina - Bahia, Brazil. Kew: Royal Botanic Gardens.

Holt, E. G. (1928) An ornithological survey of Serra do Itatiaya, Brazil. Bull. Amer. Mus. Nat. Hist. 57: 251-326.

Lara, A. C. F. and Fernandes, G. W. (1996) The highest diversity of galling insects: Serra do Cipó, Brazil. Biodiv. Lett. 3: 111-114.

Machado, A. B. M., Fonseca, G. A. B., Machado, R. B., Aguiar, L. M. S. and Lins, L. V. (1998) Livro vermelho das espécies ameaçadas de extinção da fauna de Minas Gerais. Belo Horizonte: Fundação Biodiversitas.

Maldonado-Coelho, M. and Marini, M. Â. (2000) Effects of forest fragment size and successional stage on mixed-species bird flocks in southeastern Brazil. Condor 102: 585-594.

Marini, M.Â. (2001) Effects of forest fragmentation on birds of the cerrado region, Brazil. Bird Conserv. Internatn. 11: 11-23.

Menezes, N. L. and Giulietti, A. M. (1986) Campos rupestres, paraíso botânico na Serra do Cipó. Ciência Hoje 5: 38-44.

Menezes, N. L. and Giulietti, A. M. (2000) Campos rupestres. In M. P. Mendonça and L. V. Lins, eds. Lista vermelha das espécies ameaçadas de extinção da flora de Minas Gerais. Belo Horizonte: Fundação Biodiversitas and Fundação Zoobotânica de Belo Horizonte.

Mittermeier, R. A., Myers, N., Gil, P. R. and Mittermeier, C. G. (1999) Hotspots: Earth's biologically richest and most endangered terrestrial ecoregions. Mexico City: CEMEX.

Parker III, T. A. and Rocha, O. (1991) La avifauna de Cerro San Simon, una localidad de campo rupestre aislado en el Depto. Beni, Noreste Boliviano. Ecol. Boliv. 17: 15-29.

Parrini, R., Raposo, M. A., Pacheco, J. F., Carvalhães, A. M. P., Melo-Júnior, T. A., Fonseca, P. S. M. and Minns, J. C. (1999) Birds of the Chapada Diamantina, Bahia, Brazil. Cotinga 11: 86-95.

Ribeiro, J. F. and Walter, B. M. T. (1998) Fitofisionomias do bioma cerrado. In S. M. Sano and S. P. Almeida, eds. Cerrado: ambiente e flora. Planaltina: EMBRAPA - CPAC.

Ribeiro, S. P., Carneiro, M. A. A. and Fernandes, G. W. (1998) Free-feeding insect herbivores along environmental gradients in Serra do Cipó: basis for a management plan. J. Ins. Conserv. 2: 107-118.

Ribon, R. (1998) Fatores que influenciam a distribuição da avifauna em fragmentos de Mata Atlântica nas montanhas de Minas Gerais. M.Sc. Thesis, Universidade Federal de Minas Gerais, Belo Horizonte.

Richards, D. G. (1981) Environmental acoustics and censuses of singing birds. Stud. Avian Biol. 6: 297-300.

Scott, D. A. and Brooke, M. (1985) The endangered avifauna of southeastern Brazil: a report on the BOU/WWF expeditions of $1980 / 81$ and 1981/82. Cambridge, U.K.: International Council for Bird Preservation. (Tech. Publ. 4: 115-139).

Sick, H. (1979) A voz como caráter taxonômico em aves. Bol. Mus. Nac. 294: 1-11.

Sick, H. (1997) Ornitologia Brasileira. Rio de Janeiro: Editora Nova Fronteira.

Silva, J. M. C. (1995a) Biogeographic analysis of the South American Cerrado avifauna. Steenstrupia 21: 49-67.

Silva, J. M. C. (1995b) Birds of the Cerrado Region, South America. Steenstrupia 21: 69-92.

Silva, J. M. C. (1998) Integrating biogeography and conservation: an example with birds and plants of the Cerrado region. An. Acad. Bras. Ci. 70: 881-888.

Stattersfield, A. J., Crosby, M. J., Long, A. J. and Wege, D. C. (1998) Endemic bird areas of the world, priorities for biodiversity conservation. Cambridge, U.K.: BirdLife International (Conservation Series 7). 
Wanderley, M. G. L. (1990) Diversidade e distribuição geográfica das espécies de Orthophytum (Bromeliaceae). Acta Bot. Bras. 4: 169-175.

Willis, E. O. and Oniki, Y. (1991) Avifaunal transects across the open zones of northern Minas Gerais, Brazil. Ararajuba 2: 41-58.

TADEU ARTUR DE MELO-JÚNIOR

Pós-Graduação em Zoologia, IB, Universidade Estadual Paulista, CP 199, Avenida 24-A,1515, Rio Claro, 13506-9oo,SP, Brazil.E-mail: tadeu_melojr@yahoo.com.br

\section{MARCELO FERREIRA DE VASCONCELOS}

Pós-Graduação em Ecologia, Conservação e Manejo de Vida Silvestre, ICB, Universidade Federal de Minas Gerais, C.P. 486, Belo Horizonte, 30161-970, MG, Brazil.

GERALDO WILSON FERNANDES and MIGUEL ÂNGELO MARINI

Departamento de Biologia Geral, ICB, Universidade Federal de Minas Gerais, C.P. 486, Belo Horizonte, 30161-970, MG, Brazil

Received 1 September 1999; revision accepted 17 April 2001 